\title{
Prática Mental para Pacientes com Sequelas Motoras Pós Acidente Vascular Cerebral
}

\author{
Mental Practice for Patients with Motor Sequelae After Stroke \\ Thatyana Granato de Andrade', Sabrina Kyoko de Paula Asa ${ }^{2}$
}

\begin{abstract}
RESUMO
O Acidente Vascular Cerebral (AVC) é uma doença incapacitante de alta incidência, que representa um problema de saúde pública em muitos países. Entre as diversas abordagens fisioterapêuticas disponíveis atualmente, a prática mental (PM) tem sido apontada como um dos métodos para aquisição e melhora das habilidades motoras. Método. Revisão de literatura, realizada durante os meses de agosto e setembro de 2009. Foram incluídos artigos científicos publicados nos anos de 1966 a 2009 encontrados nas bases de dados PUBMED, EMBASE e SCIELO, com os descritores stroke, rehabilitation, mental practice, acidente vascular cerebral, reabilitação, prática mental, e aqueles encontrados por busca manual das referências citadas nos artigos encontrados. Resultados. Foram encontrados vinte e um artigos, dos quais seis foram excluídos. A idade média dos participantes dos estudos foi de 60,8 anos, sendo estes predominantemente do gênero masculino. Houve grande variaçáo com relação à metodologia aplicada, tarefa motora treinada e escalas de avaliação selecionadas para aplicação. Conclusáo. A PM pode ser considerada um recurso terapêutico adicional, disponível para aplicação em pacientes com características específicas. Apesar de resultados satisfatórios terem sido demonstrados, os estudos publicados até o momento não fornecem evidências científicas suficientes para sustentar de forma confiável sua aplicação.
\end{abstract}

Unitermos. Acidente Vascular Cerebral, Prática Mental, Reabilitação, Fisioterapia.

Citaçáo. Andrade TG, Asa SKP. Prática Mental para Pacientes com Sequelas Motoras Pós Acidente Vascular Cerebral.

\begin{abstract}
Stroke is an incapacitating disease of elevated incidence in adults, and represents a public health problem in many countries. Amongst the many therapeutic approaches available nowadays for physical therapeutic intervention, mental practice has been pointed as a method for acquisition and improvement of motor abilities. Method. Literature review during the period of August and September 2009. Were included scientific articles published from 1966 to 2009 found in PUBMED, EMBASE and SCIELO databases, with the following keywords: stroke, rehabilitation, mental practice, acidente vascular cerebral, reabilitaçâo, prática mental, and others found by manual searching of the references cited in the articles found. Results. Twenty one articles were found, from which six were excluded. The average age of the participants was 60,8 years and they were predominantly men. There was wide variation in relation to the protocol used, type of motor skill focused on and type of scales used for evaluation. Conclusion. Mental practice can be considered an additional therapeutic approach, available for application in patients with specific caractheristics. Despite satisfactory results been demonstrated, articles published until the present moment do not show scientific evidence to sustain the application of mental practice in a reliable way.
\end{abstract}

Keywords. Stroke, Mental Practice, Rehabilitation, Physical Therapy.

Citation. Andrade TG, Asa SKP. Mental Practice for Patients with Motor Sequelae After Stroke.

\footnotetext{
Trabalho realizado na Associaçáo de Assistência à Criança Deficiente (AACD), Sáo Paulo-SP, Brasil.

1. Fisioterapeuta, Especialista em Fisioterapia em Neurologia- Hospital Israelita Albert Einstein, Fisioterapeuta da Associação de Assistência à Criança Deficiente (AACD), São Paulo-SP, Brasil.

2. Fisioterapeuta, Especialista em Intervençấo Fisioterapêutica nas Doenças Neuromusculares - UNIFESP, Mestranda em Neurociências e Comportamento - Instituto de Psicologia - USP, Fisioterapeuta da Associaçáo de Assistência à Criança Deficiente (AACD), São Paulo-SP, Brasil.
}

Endereço para correspondência: Thatyana G Andrade

R. Fernandes Moreira, 582, ap 142 Ch Santo Antônio CEP 04716-001, São Paulo-SP, Brasil. Tel.: 5183-6041

E-mail: a_thaty@hotmail.com 


\section{INTRODUÇÃO}

Prática mental (PM), também chamada de ensaio mental ou simbólico, consiste no método de treinamento em que a reprodução interna de um determinado evento é repetida extensivamente com a intençấo de aprender uma nova habilidade ou melhorar o desempenho de uma tarefa já conhecida. Por outro lado, o processo de imaginar um movimento, uma ou algumas vezes, é chamado de imaginação motora. Dessa forma, imaginação motora se refere a um processo cognitivo específico, enquanto PM está relacionada a um método de treinamento que utiliza vários processos cognitivos, incluindo a imaginação motora ${ }^{1}$.

Resultados de estudos de psicologia nos esportes ${ }^{2}$ e no aprendizado de tarefas ${ }^{3,4}$ mostram que a prática mental com imaginação motora melhora o desempenho dos indivíduos quando comparado às condições sem prática, embora em menor extensão quando comparado à somente a prática física. Isto se deve ao desencadeamento de processos neurais, com ativação de áreas cerebrais (córtex frontal, córtex pré-motor, córtex motor primário, cerebelo, putâmen, giro frontal inferior, área motora suplementar) semelhantes àquelas ativadas durante o planejamento e execuçáo do movimento ${ }^{3,5,6}$. Estes achados sugerem que a prática mental com imaginação motora pode ser efetiva na melhora do aprendizado de tarefas motoras ${ }^{7}$, esteja o indivíduo em um estágio inicial de aprendizagem ou não.

Ao analisar a literatura sobre PM, observa-se que apenas uma pequena quantidade de tentativas de aplicála no contexto da reabilitação foi realizada. Uma possível razão para a falta de evidências experimentais é que as bases teóricas e práticas sobre quando e como implementar a PM na reabilitação física ainda não estão bem estabelecidas. Além disso, os instrumentos para mensurar a melhora no desempenho podem ser insensíveis para o tipo e a magnitude das mudanças que podem ser observadas com a $\mathrm{PM}^{7}$.

Visto que a incidência do acidente vascular cerebral (AVC) é crescente e que aproximadamente 60\% dos indivíduos acometidos apresentam sequelas motoras crônicas $^{6}$ e consequentemente deveriam ser inseridos em um programa de reabilitação, este estudo tem como objetivo verificar de que forma a PM tem sido aplicada, quais os resultados demonstrados e, se pelas evidências acumuladas em literatura, ela pode ser um recurso utilizado na reabilitação física de pacientes adultos que sofreram uma lesão neurológica decorrente de AVC.

\section{MÉTODO}

Para identificação e caracterização dos estudos clínicos que utilizaram a PM como recurso terapêutico em pacientes com AVC, utilizou-se de revisão bibliográfica, realizada durante os meses de agosto e setembro de 2009. Foram incluídos artigos científicos publicados nos anos de 1966 a 2005 encontrados nas bases de dados PUBMED, EMBASE e SCIELO, que utilizaram descritores na língua inglesa stroke, rehabilitation e mental practice e em português acidente vascular cerebral, reabilitação e prática mental, e aqueles encontrados por busca manual das referências citadas nos artigos encontrados.

Após a revisão bibliográfica foram excluídos os estudos que tratavam de pacientes com outros diagnósticos além de AVC, ou que descreviam apenas protocolos de intervenção, sem resultados sobre a intervenção aplicada.

\section{RESULTADOS}

Através do levantamento descrito na metodologia, foram encontrados vinte e um artigos, dos quais cinco foram excluídos por descreverem apenas protocolos de intervenção, sem resultados; e um foi excluído por agrupar pacientes com outros diagnósticos, além dos pacientes com AVC. A idade média dos participantes dos estudos foi de 60,8 anos, sendo que estes eram predominantemente do gênero masculino.

A caracterização dos estudos quanto ao tipo de estudo, idade média e tempo de lesão dos pacientes estudados, avaliaçóes padronizadas e escalas utilizadas, tipo de intervenção e resultados obtidos está demonstrada na Tabela 1. 
Tabela 1

Caracterização dos estudos quanto ao tipo de estudo, idade média e tempo de lesão dos pacientes estudados, avaliaçöes padronizadas e escalas utilizadas, tipo de intervenção e resultados obtidos

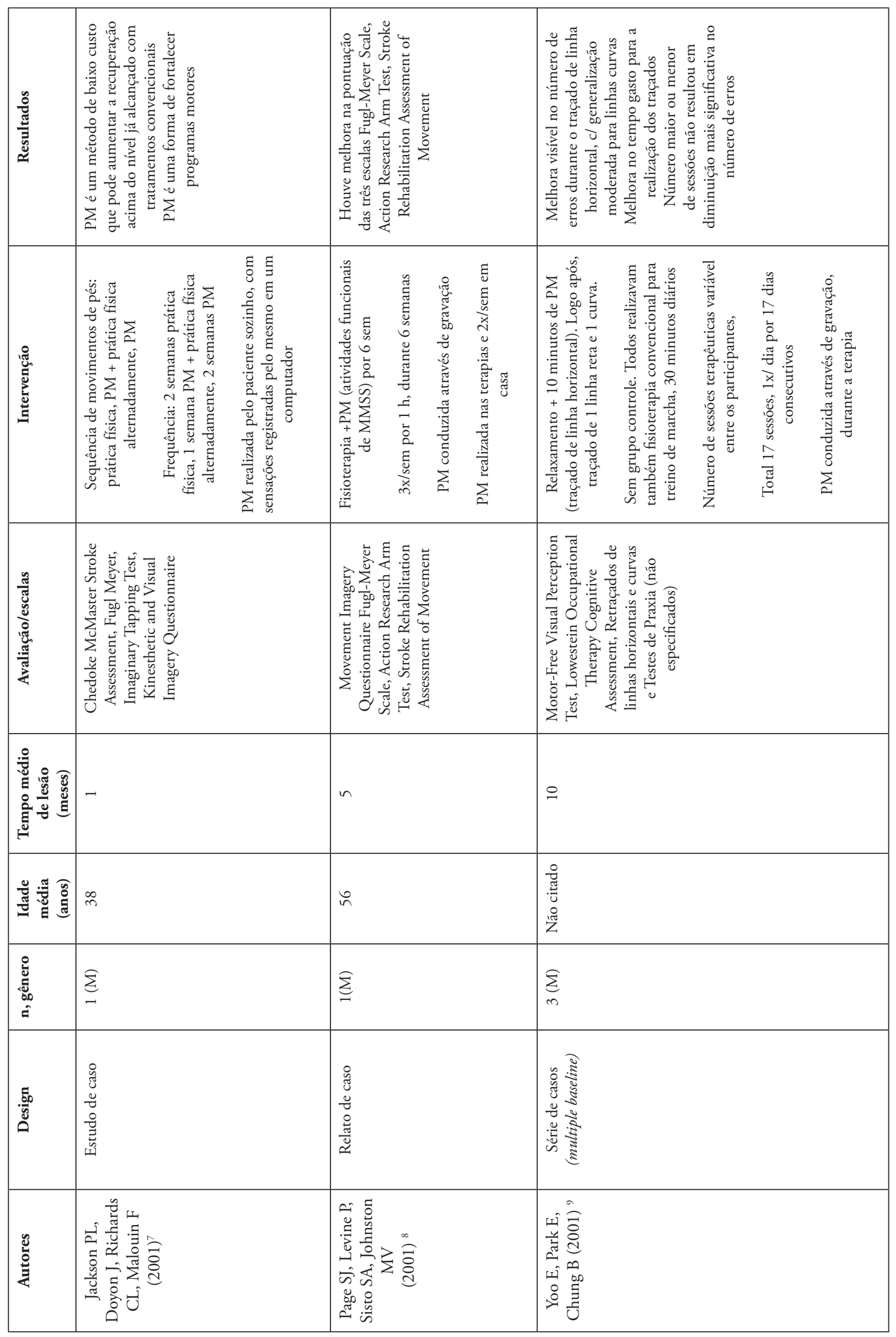


Tabela 1

Continuação.

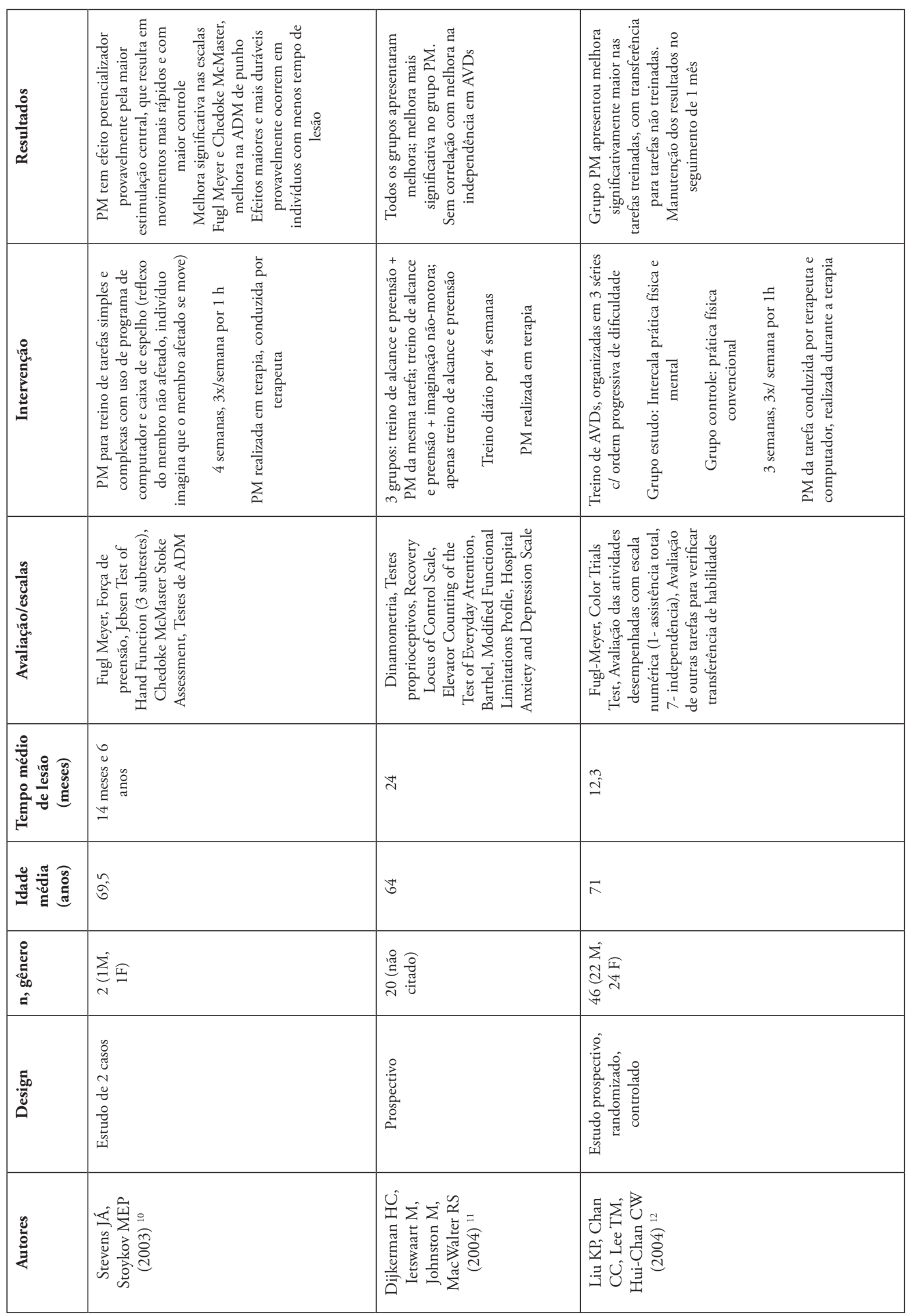


Tabela 1

Continuação.

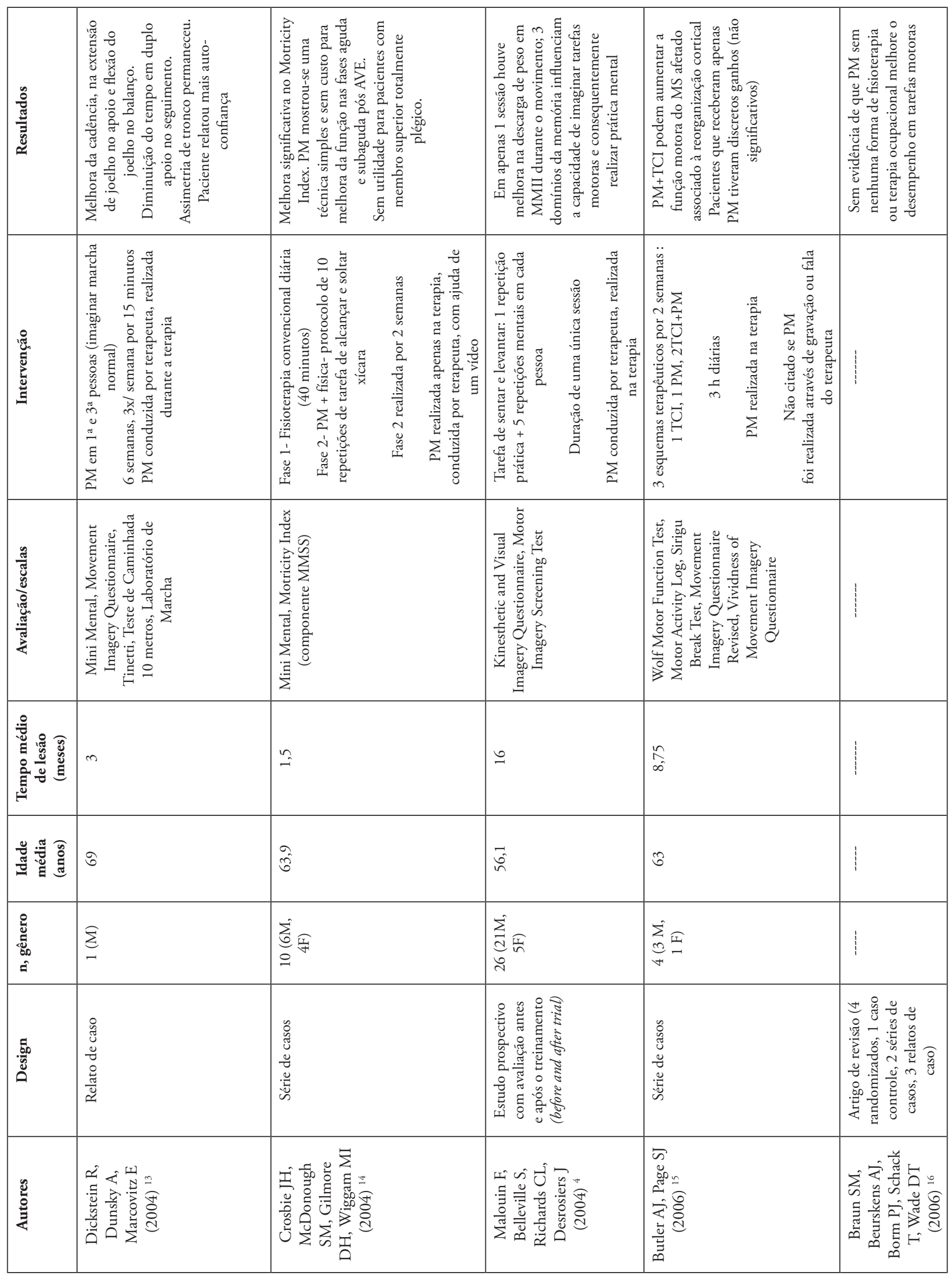


Tabela 1

Continuaçấo.

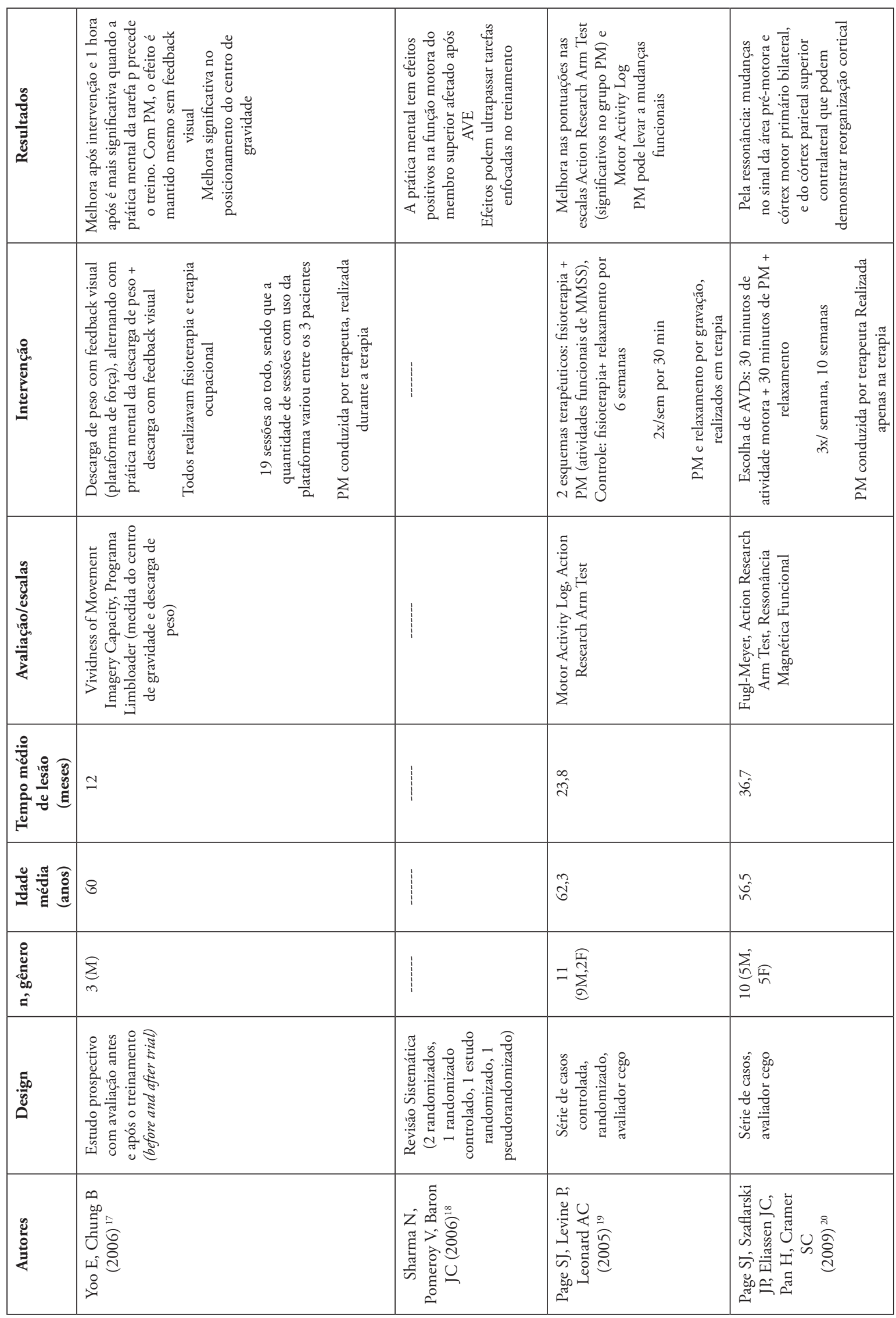

M: masculino; F: feminino; MMSS: membros superiores; MMII: membros inferiores TCI: Terapia por Contensão Induzida; AVDs: atividades de vida diária 


\section{DISCUSSÃO}

O Acidente Vascular Cerebral é uma patologia incapacitante de alta incidência, que representa um problema de saúde pública em muitos países do mundo ${ }^{16}$. Entre as diversas abordagens terapêuticas disponíveis atualmente para a intervenção fisioterapêutica no AVC, a prática mental tem sido apontada como um dos métodos para aquisição e melhora das habilidades motoras nos pacientes acometidos ${ }^{17}$.

Os estudos levantados demonstraram vantagens à aplicação da prática mental no AVC. Em geral, concluíram que a técnica tem efeitos positivos na função motora do paciente hemiparético. Em relação ao membro superior, são descritas mudanças como a melhora no traçado de linhas horizontais e curvas com lápis ${ }^{9}$, melhora na amplitude de movimento de punho ${ }^{10}$ e melhora na força de preensão ${ }^{11}$. Com relação aos membros inferiores e à marcha, são descritas melhora na descarga de peso dinâmica no lado hemiparético ${ }^{4}$, melhora no posicionamento do centro de gravidade ${ }^{17}$, melhora da cadência, extensão do joelho no apoio e flexão do joelho no balanço; e diminuição do tempo em duplo apoio ${ }^{13}$. Descrevem-se também melhoras funcionais, na independência nas atividades de vida diária e no controle muscular do lado hemiparéti$\mathrm{co}^{10,12}$, com melhora na pontuação em diversas escalas motoras. Estas alterações estão descritas na Tabela 1.

Os artigos demonstram que os efeitos benéficos da PM podem ultrapassar as tarefas enfocadas no tratamento, tornando possível a generalização para outras habilidades. Pode contribuir, também, para a reorganização cortical. Estudos que avaliaram a imaginação motora através da ressonância magnética funcional ou da estimulação magnética transcraniana, isolando a atividade muscular através da eletromiografia, demonstraram que áreas cerebrais como o córtex motor primário, área motora suplementar e área pré-motora são ativadas mesmo na ausência da ação muscular ${ }^{3}$.

O cerebelo, que está altamente relacionado ao córtex sensório-motor ao ser responsável pela retroalimentação sensorial do movimento, adequando a transferência da representação interna do movimento às condiçóes físicas do ambiente externo, também é ativado durante a imaginação motora ${ }^{3}$. Desta forma, constitui um instrumento que pode compensar a falta de retroalimentação sensorial no paciente, sendo uma maneira de reforçar programas motores que objetivam a aprendizagem de tarefas ${ }^{1,12,15,18}$.

A área motora suplementar e o córtex pré-motor também são ativados durante a imaginação motora, como comprovado em vários estudos ${ }^{3,21,22}$. Os neurônios da área motora suplementar estão envolvidos na preparaçẫo dos movimentos, dessa forma os aspectos preparatórios do movimento podem estar diretamente relacionados com a imaginação motora. Em relação ao córtex pré-motor, diferentes estratégias de imaginação, como imaginar o próprio movimento ou imaginar o movimento no espaço levam à ativação de diferentes áreas desta regiāo $0^{3,22}$.

Outra área ativada durante a imaginação motora é a região superior do lobo parietal, que permanece ativa durante atividades que envolvem aspectos espaciais como movimentos com joystick ou trajetórias de movimento ${ }^{3}$.

A neuroimagem demonstra também que durante a imaginação motora são fortemente ativadas as vias motoras descendentes, com excitabilidade corticoespinal bastante semelhante quando a prática mental é comparada à prática física. Há também similaridades quanto à modulação no tempo de duração da tarefa e na resposta moduladora autonômica, de forma que a imaginação motora e a prática real realmente engajem sistemas cerebrais semelhantes ${ }^{22}$.

Há relatos de que a realização da prática mental contribuiu também para que os pacientes se sentissem mais motivados e autoconfiantes, com maior segurança antes de desempenhar determinadas atividades ${ }^{13,17}$.

No entanto, é uma abordagem que tem restriçóes à aplicação dependendo do comprometimento do paciente: não é aplicável para pacientes apráxicos e heminegligentes $^{17}$ e naqueles que não são capazes de imaginar o movimento ou têm lentidão no processamento da imaginação motora $^{1,11}$. Pacientes com lesôes nas regiôes parietais e pré-frontal esquerda não são capazes de imaginar uma tarefa motora. Lesôes parietais bilaterais resultam em uma completa falta de percepção da execução do movimento durante a imaginação motora ${ }^{3}$.

Assim, mostra-se necessário o uso de questionários para avaliar a capacidade de imaginação motora do paciente, o que não foi feito em todos os estudos. Existem questionários validados, descritos na Tabela 1 , como por exemplo o Kinesthetic and Visual Imagery Questionnaire ${ }^{7}$, 
Movement Imagery Questionnaire ${ }^{13}$, Motor Imagery Screening Test ${ }^{4}$ e Vividness of Movement Imagery Questionnaire ${ }^{15}$. Mesmo naqueles que concluíram boa capacidade de imaginação em seus sujeitos, há sempre a incerteza e incapacidade de controlar a execuçâo do indivíduo, tanto em frequência quanto à forma como ele imagina os movimentos relacionados à tarefa motora ${ }^{18}$.

Também foi levantada a incerteza quanto aos efeitos que podem ocorrer em pacientes muito comprometidos (por exemplo, plegia total em membro superior) ${ }^{14}$ e a necessidade de que o paciente já tenha alguma habilidade prévia em realizar a tarefa a ser treinada com a PM, ou seja, alguma representação interna do movimento, para que os resultados sejam satisfatórios ${ }^{6}$.

Todos os trabalhos levantados estudaram um número de sujeitos insuficiente para traçar respostas conclusivas aos questionamentos sobre o uso da PM no AVC. O número de publicaçoos é pequeno e entre elas, há estudos sem grupo controle e muitos relatos de caso, como demonstra a Tabela 1. Além disso, a metodologia dos estudos é extremamente diversa: cada estudo utiliza-se de um tipo de tarefa motora e seleciona escalas de avaliação diferentes, o que torna difícil a comparação entre eles.

Muitos estudos não descreveram o perfil dos pacientes quanto ao tipo e local de lesão encefálica, e entre eles houve grande variação quanto ao tempo de lesão dos pacientes. A evolução de pacientes com AVC na fase aguda, subaguda e crônica é muito diferente e não se determinou qual seria o período ideal para início do treinamento ${ }^{18}$. Alguns autores defenderam que os resultados seriam mais promissores na fase inicial de lesão (menos de seis meses) ${ }^{10}$, enquanto para outros os resultados seriam altamente satisfatórios em pacientes crônicos, que já atingiram um platô de evolução da capacidade motora ${ }^{1,19}$.

A forma com que a prática mental era desempenhada também foi muito variável entre os estudos. A frequência de aplicação variou de apenas uma sessão a três sessões semanais durante períodos de até seis semanas, alternadamente com sessôes de fisioterapia motora ou não. O procedimento foi realizado em ambiente ambulatorial ou doméstico, através de orientação verbal do terapeuta, uso de espelhos, instruções contidas em fitas gravadas, ou com auxílio de computador. Foi apontado por relatos de pacientes que o uso de uma fita gravada, ao invés da instrução verbal do terapeuta, pode torná-los mais passivos e desconcentrados, por conta da repetição das instruçôes com as mesmas palavras, entonação e duração?. Alguns estudos incluíram sessóes de relaxamento durante a intervenção. Permanece então, uma ampla gama de modelos de intervenção a serem testados por terapeutas em sua prática clínica.

A imaginação motora parece ter função importante na preparação e treinamento do movimento. Uma possibilidade da utilização da imaginação motora na prática clínica é fazer com que o indivíduo não considere as lesões que possui durante o treinamento e planejamento de movimentos mais complexos ${ }^{3}$.

O treinamento mental parece ser uma técnica complementar porém não substitui a execução motora dos movimentos, capaz de proporcionar efeitos adicionais ao treinamento motor pela maior estimulação central ${ }^{1,10,12,20}$, além de diversificá-lo. Pode ser aplicado com segurança, e dispensa instalaçóes especiais e equipamentos, sendo um recurso simples e de baixo custo ${ }^{11,14}$. Pelo fato de poder ser iniciado precocemente, mesmo em um estado de plegia, pode-se pensar na aplicação em ambiente hospitalar, com pacientes responsivos, ainda que instáveis. Pensando na realidade dos programas de reabilitação motora da rede pública em nosso país, que tem número restrito de sessôes semanais, é uma técnica interessante, pois o paciente pode ser orientado adequadamente e realizar o treinamento sozinho. Pode ser considerado um complemento para pacientes com pouca mobilidade funcional e alto gasto energético, já que evita o estresse articular e físico decorrente de prática física excessiva ${ }^{12,14}$.

\section{CONCLUSÃO}

Concluímos que a prática mental em pacientes com sequelas motoras após AVC pode ser considerada como um complemento terapêutico disponível para aplicação em pacientes com características específicas. Apesar de resultados satisfatórios terem sido demonstrados, os estudos publicados até o momento não fornecem evidências científicas suficientes para sustentar de forma confiável sua aplicação. 


\section{REFERÊNCIAS}

1.Jackson PL, Lafleur MF, Malouin F, Richards C, Doyon J. Potential role of mental practice using motor imagery in neurologic rehabilitation. Arch Phys Med Rehabil 2001;82:1133-41.

http://dx.doi.org/10.1053/apmr.2001.24286

2.Teixeira LA. Aprendizagem de habilidades motoras na ginástica artística. Em: Nunomura M \& Nista-Piccolo VL (Eds), Compreendendo a ginástica artística. São Paulo, Phorte. 2004, p.77-106.

3.Lotze M, Halsband U. Motor imagery. J Physiol 2006;99:386-95.

4.Malouin F, Belleville S, Richards CL, Desrosiers J, Doyon J. Working memory and mental practice outcomes after stroke. Arch Phys Med Rehabil 2004;85:177-83.

http://dx.doi.org/10.1016/S0003-9993(03)00771-8

5.Li CR. Impairment of motor imagery in putamen lesions in humans. Neuroscience Letters 2000;287:13-6.

http://dx.doi.org/10.1016/S0304-3940(00)01164-2

6.Vries SD, Mulder T. Motor imagery and stroke rehabilitation: a critical discussion. J Rehabil Med 2007;39:5-13.

http://dx.doi.org/10.2340/16501977-0020

7.Jackson PL, Doyon J, Richards CL, Malouin F. The efficacy of combined physical and mental practice in the learning of a foot-sequence task after stroke: a case report. Neurorehabil Neural Repair 2004;18:106-11.

http://dx.doi.org/10.1177/0888439004265249

8.Page SJ, Levine P, Sisto SA, Johnston MV. Mental practice combined with physical practice for upper-limb motor deficit in subacute stroke. Phys Ter 2001;81:1455-62.

9.Yoo E, Park E, Chung B. Mental practice effect on line-tracing accuracy in persons with hemiparetic stroke: a preliminary study. Arch Phys Med Rehabil 2001;82:1213-8.

http://dx.doi.org/10.1053/apmr.2001.25095

10.Stevens JA, Stoykov MEP. Using motor imagery in the rehabilitation of hemiparesis. Arch Phys Med Rehabil 2003;84:1090-2.

http://dx.doi.org/10.1016/S0003-9993(03)00042-X

11.Dijkerman HC, Ietswaart M, Johnston M, MacWalter RS. Does motor imagery training improve hand function in chronic stroke patients? A pilot study. Clin Rehabil 2004;18:538-49.

http://dx.doi.org/10.1191/0269215504cr769oa

12.Liu KP, Chan CC, Lee TM, Hui-Chan CW. Mental imagery for promoting relearning for people after stroke: a randomized controlled trial. Arch Phys Med Rehabil 2004;85:1403-8.

http://dx.doi.org/10.1016/j.apmr.2003.12.035

13.Dickstein R, Dunsky A, Marcovitz E. Motor imagery for gait rehabilitation in post-stroke hemiparesis. Phys Ter 2004;84:1167-77.

14.Crosbie JH, McDonough SM, Gilmore DH, Wiggam MI. The adjuntive role of mental practice in the rehabilitation of the upper limb after hemiplegic stroke: a pilot study. Clin Rehabil 2004;18:60-8.

http://dx.doi.org/10.1191/0269215504cr702oa

15.Butler AJ, Page SJ. Mental practice with motor imagery: evidence for motor recovery and cortical reorganization after stroke. Arch Phys Med Rehabil 2006;87(12 Suppl 2):S2-11.

http://dx.doi.org/10.1016/j.apmr.2006.08.326

16.Braun SM, Beurskens AJ, Borm PJ, Schack T, Wade DT. The effects of mental practice in stroke rehabilitation: a systematic review. Arch Phys Med Rehabil 2006;87:842-52.

http://dx.doi.org/10.1016/j.apmr.2006.02.034

17.Yoo E, Chung B. The effect of visual feedback plus mental practice on symmetrical weight-bearing training in people with hemiparesis. Clin Rehabil 2006;20:388-97.

http://dx.doi.org/10.1191/0269215506cr962oa

18.Sharma N, Pomeroy VM, Baron JC. Motor imagery: a backdoor to the motor system after stroke? Stroke 2006;37:1941-52.

http://dx.doi.org/10.1161/01.STR.0000226902.43357.fc

19.Page SJ, Levine P, Leonard AC. Effects of mental practice on affected limb use and function in chronic stroke. Arch Phys Med Rehabil 2005;86:399-402. http://dx.doi.org/10.1016/j.apmr.2004.10.002

20.Page SJ, Szaflarski JP, Eliassen JC, Pan H, Cramer SC. Cortical plasticity following motor skill learning during mental practice in stroke. Neurorehabil Neural Repair. 2009;23:382-8. http://dx.doi.org/10.1177/1545968308326427

21.Lacourse MG, Turner JA, Randolph-Orr E, Schandler SL, Cohen MJ. Cerebral and cerebellar sensorimotor plasticity following motor imagery-based mental practice of a sequential movement. J Rehabil Res Dev 2004;41:505-24. http://dx.doi.org/10.1682/JRRD.2004.04.0505

22.Mulder T. Motor imagery and action observation: cognitive tools for rehabilitation. J Neural Transm 2007;114:1265-78.

http://dx.doi.org/10.1007/s00702-007-0763-z 